\title{
A Load Balance Based On-Demand Routing Protocol for Mobile Ad-Hoc Networks
}

\author{
Liqiang Zhao ${ }^{1}$, Xin Wang ${ }^{2}$, Azman Osman Lim ${ }^{3}$, and Xiangyang Xue \\ ${ }^{1}$ School of Software, Fudan University, Shanghai, China \\ ${ }^{2}$ Dept. of Computer Science and Engineering, \\ Fudan University, Shanghai, China \\ \{zhaolq, xinw, xyxue\} @fudan.edu.cn \\ ${ }^{3}$ Dept. of Computer Science, Kyoto University, Kyoto, Japan \\ alvin1973@hotmail.co.jp
}

\begin{abstract}
Since the existing ad hoc routing protocols lack of load balance capabilities, they often fail to provide satisfactory performance in the presence of a large volume of traffic. In this paper, we propose a new load balance mechanism and a novel bandwidth estimation method for ad hoc on-demand routing protocols. Destination chooses the optimal path via the route information carried by RREQ, whilst congested intermediate nodes dropped RREQs to avoid RREQ storm. Simulation shows that our new scheme improves packet delivery ratio, reduces end-to-end latency and decreases routing overhead.
\end{abstract}

\section{Introduction}

A Mobile Ad hoc Network (MANET) is a self-configuring network of mobile hosts connected by wireless links. Due to its unique character of self-organization, quick deployment and infrastructure-free, MANET has a wide range of applications which include battlefield command and control, emergency disaster relief, mine site operations.

Currently, on-demand routing protocol is the dominant routing protocol in MANET. As the typical on-demand routing protocols, AODV [7] and DSR [2] select the shortest routes as the optimum routes. However, due to the special characters of ad hoc network, many researches realize that the shortest route may not be the best criteria of route selection $[9,14]$.

Meanwhile, researchers have been focusing on network load balance. Due to the constrained ad hoc network bandwidth resource, routing protocols are required to properly distribute the traffic flow over the network. Otherwise, the overloaded nodes may cause network congestion and long delay. In considering the significance of network traffic balance, we propose a load balance mechanism, which can virtually be applied to any on-demand routing protocol. In this paper, we combine this mechanism with AODV, forming a new routing protocol called load balance based AODV (LBB-AODV).

The rest of this paper is organized as follows: Section 2 introduces some related work about load balance routing in ad hoc network. In section 3, we propose a novel 
residual bandwidth estimation model and a new load balance mechanism, both of which are incorporated into the routing protocol LBB-AODV. Section 4 is the simulation and analysis. Finally, section 5 concludes this paper.

\section{Related Work}

Multiple paths schemes, such as [10] and [13], are fully exploited to provide better load-balancing capabilities. The underlying idea of the scheme is to distribute the traffic among multiple paths, which are maintained at nodes and used for routing. However, maintaining alternative paths requires more routing table space and computational overhead while selecting the best route among several discovered paths [11]. Moreover, multiple routes schemes are effective only if the alternate multi-path are disjoint, which is difficult to achieve in MANET [6]. Gan jali et al. [8] further demonstrates that the load distribution of multi-path routing is almost the same as that of single path routing.

DLAR [3], a single-path load balance mechanism, is proposed. The RREQ records queue occupancy information of each node it traverses. The destination chooses the optimal path based on the load information kept in RREQs. However, to utilize the most up-to-date load information, DLAR prohibits intermediate nodes from replying to RREQ, which may result in RREQ storm when there are a large number of nodes in the mobile ad hoc network.

In [4], a new scheme is presented that each node forward RREQs selectively according to the load status of the node, preventing the new routes set up through overloaded nodes. This may alleviate the possibility of RREQ storm, but since it works in a fully distributed manner, the route selected may not be the best one due to the lack of comparison of candidate routes.

\section{Load Balance Based-AODV}

LBB-AODV is an enhanced version of AODV based on load balance, which enable nodes to forward RREQ selectively during route discovery stage. When a node receives route request, it first checks its available bandwidth. The node forwards the RREQ only if it is not CONGESTED. After the destination receives all the route information via RREQ, it picks the best route. The detail description of bandwidth estimation and LBB-AODV are presented as follows.

\subsection{Bandwidth Estimation}

To guarantee the network load balance, it is necessary to know each node's available bandwidth. The end-to-end throughput is a concave parameter [5], which is determined by the bottleneck bandwidth of the intermediate nodes along the route. Therefore, estimating available bandwidth of a specific route can be simplified into finding the minimal residual bandwidth available among the nodes in the route.

We propose a simple and effective available bandwidth estimation model in ad hoc network. In the new scheme, each the node is set to promiscuous mode, so it can 
perceive any frame sent by its neighboring nodes. Every node accumulates the size of the perceived frames within a predefined period of time, and adds it to the size of frames the node sends itself during the time, the throughput of the small area centering the node within the time window is gained. By examining the activities of both the node itself and its surrounding neighbors, we are able to obtain a good approximation of the bandwidth usage. The available bandwidth of a node is:

$$
\mathrm{B}_{\text {avail }}=\mathrm{B}_{\text {raw }}-\mathrm{B}_{\text {tran }}-\mathrm{B}_{\text {recv }}
$$

$\mathrm{B}_{\text {avail }}$ is the available bandwidth of the node, while $B_{\text {raw }}$ is ad hoc network pure channel bandwidth. $\mathrm{B}_{\text {tran }}$ is the consumed bandwidth of the node by sending frames, including data and routing control frames and 802.11MAC layer RTS/CTS/ACK control frames. $\mathrm{B}_{\text {recv }}$ is the bandwidth took up by neighboring nodes.

$\mathrm{B}_{\text {tran }}$ and $\mathrm{B}_{\text {recv }}$ can be represented as follows:

$$
\begin{aligned}
& \mathrm{B}_{\text {tran }}=\mathrm{TH}_{\text {tran }} / \mathrm{t} \\
& \mathrm{B}_{\text {recv }}=\mathrm{TH}_{\text {recv }} / \mathrm{t}
\end{aligned}
$$

Here, $\mathrm{t}$ is the predefined period of time, when $\mathrm{TH}_{\text {tran }}$ and $\mathrm{TH}_{\text {recv }}$ are the size of frames transmitted and received, respectively, within t by the node.

Taking into consideration the channel fading, physical error, the frame collision , as well as the burst nature of control packets overhead, factor $\theta \in(0,1)$ is used to adjust $\mathrm{B}_{\text {avail. }}$ Combine function (1), (2) and (3), we get:

$$
\mathrm{B}_{\text {avail }}=\left(\mathrm{B}_{\text {raw }}-\left(\mathrm{TH}_{\text {tran }}+\mathrm{TH}_{\text {recv }}\right) / \mathrm{t}\right) \cdot \theta
$$

At the beginning of the simulation, $\mathrm{TH}_{\text {tran }}$ and $\mathrm{TH}_{\text {recv }}$ of every node are set to 0 while the timeout is set to $\mathrm{t}$. When it timeouts, the available bandwidth can be calculated by (4). Store it in the cache. Then reset $\mathrm{TH}_{\text {tran }}$ and $\mathrm{TH}_{\text {recv }}$ to 0 , calculate the bandwidth of next period.

The calculation of the period time $t$ is critical; it should not be too big or too small. If it is set too small, there might be no node to send packet during that short period. In this case, the function only reflects transit bandwidth utilization, and it might not be the stable bandwidth consumption condition. Moreover, short period means frequent calculation, which will consume a batch of CPU time and battery power. If $t$ is set too big, because of the movement of the node and change of link state, the function might not be able to reflect the real bandwidth usage. Moreover, $t$ should be proportional to density of the whole network, since the higher the node's density is, the higher chance of packet collision, and the larger t should be [12].

To smooth bandwidth estimation, we define a smoothing constant $\beta \in(0,1)$. Suppose the last bandwidth is $B_{\text {avail(n-1) }}$ and the bandwidth measured in the current sampling time window is $B_{\text {avail }}$. Then, the current bandwidth $B_{\text {avail(n) }}$ is given as:

$$
\mathrm{B}_{\text {avail(n) }}=\beta \cdot \mathrm{B}_{\text {avail(n-1) }}+(1-\beta) \cdot \mathrm{B}_{\text {avail }}
$$




\subsection{LBB-AODV}

For our purpose, a new field $B_{\min }$ is added in RREQ format to record the minimum bandwidth along the path it traverses. When a source node decides to launch a RREQ, it first estimates its bandwidth using mechanism described above, and records it in $\mathrm{B}_{\text {min. }}$.

When an intermediate node first receives a RREQ, it will collect its load information in order to define present network condition. According to the network condition, routing layer determines whether to forward or just drop the RREQ. The network has two conditions: CONGESTION and NORMAL, which is defined by ratio of the current available bandwidth $\left(\mathrm{B}_{\text {avail }}\right)$ and the total channel bandwidth $\left(\mathrm{B}_{\text {raw }}\right)$ with $\varphi$ being the congestion factor:

$$
\begin{aligned}
& \text { CONGESTION: } \mathrm{B}_{\text {avail }} / \mathrm{B}_{\text {raw }} \leq \phi \\
& \text { NORMAL: } \mathrm{B}_{\text {avail }} / \mathrm{B}_{\text {raw }}>\phi
\end{aligned}
$$

When an intermediate node is in the CONGESTION mode, the node will no longer process any route request; it simply discards RREQ, making itself impossible to be an intermediate node for other traffic. Each node begins to allow additional traffic flows whenever its congested status is dissolved.

A new field $B_{t}$ is added to the route table of each node to record the so-fardiscovered-biggest route bandwidth from the source to the node. When the node is in NORMAL condition, if it is the first time receiving RREQ from the source node with the broadcast $\mathrm{id}, \mathrm{B}_{\mathrm{t}}$ will be set as $\mathrm{B}_{\min }$ for the corresponding entry in the route table. Then compare $\mathrm{B}_{\min }$ and the node's available bandwidth, keep the smaller one in the $\mathrm{B}_{\min }$ and forward RREQ. If the node has already received RREQ from the same source node with the same broadcast id, it compares $B_{\min }$ with the corresponding $B_{t}$. If the former is not bigger, simply discard the RREQ. Otherwise, update the route table entry to replace $B_{t}$ with $B_{\min }$ and redirect the previous node index to the node where the RREQ is from. Finally, discard the RREQ rather than forwarding it to avoid RREQ storm.

After receiving the first RREQ, the destination waits for an appropriate amount of time to learn all possible routes. After receiving duplicate RREQ from different previous nodes, the destination chooses the route with the largest $B_{\min }$ and sends RREP.

By rejecting route request at the individual congested nodes, the network is able to reduce the large volume of routing requests and alleviate the burden brought by the broadcasting. On the other hand, choosing the best route at the destination node provide the best route along light-loaded nodes.

When the local congestion occurs, since the congested nodes refuse to forward routing request, it is possible to fail the route discovery. To cope with this scenario, after the first round route discovery failure, the original AODV will be effective to find the route from the second round. 


\section{Numerical Simulation}

In this section, we evaluate the performance of LBB-AODV compared to the conventional AODV. The simulation environment, metrics and results are presented.

\subsection{Simulation Environment}

We evaluate LBB-AODV and AODV by using the network simulator ns- 2 . In ns-2 simulator, we use the default value of AODV to demonstrate a fair comparison of LBB-AODV. The distribution coordination function (DCF) of IEEE 802.11 standard is used as the MAC protocol with a data rate of $2 \mathrm{Mbps}$.

A network consists of 100 nodes with $250 \mathrm{~m}$ transmission range spread randomly over an area of $2400 \mathrm{~m} \times 800 \mathrm{~m}$. The nodes uniformly choose velocity from $0 \mathrm{~m} / \mathrm{s}$ to $5 \mathrm{~m} / \mathrm{s}$ following the random-waypoint model. Each simulation runs for $200 \mathrm{~s}$ with pause time set to $50 \mathrm{~s}$.

Source and destination node pairs are randomly selected and each source generates CBR packets with packet size of 512 bytes. We use 40 traffic flows and gradually increase the data rate of each flow from 3 to 9 packets/second (or 480 to $1440 \mathrm{~Kb} / \mathrm{s}$ ). The traffic load represents the combined throughput of the data sources. By adjusting the frequency of sources sending packets, we eventually control the traffic load of the network.

After several simulation comparisons, we choose the following parameter values used for LBB-AODV, which are specified in Table 1.

Table 1. Parameters Used for LBB-AODV

\begin{tabular}{|c|c|}
\hline Parameter & Value \\
\hline $\mathrm{t}$ & $1 \mathrm{~s}$ \\
\hline$\theta$ & 0.9 \\
\hline$\beta$ & 0.2 \\
\hline$\varphi$ & 0.1 \\
\hline
\end{tabular}

\subsection{Simulation Metrics}

The following three metrics are considered to evaluate the performance of the protocols:

1. Packet delivery ratio - the ratio of the total number of data packets received by destinations over the total number of data packets transmitted by sources. It captures the network throughput.

2. End-to-end delay -the average of delays for all received data packet from the sources to destinations. It includes all possible delays caused by buffering during route discovery, queuing at the interface queue, retransmission delays at the medium access control layer, and propagation and transfer time.

3. Normalized routing overhead - the total number of routing control packets (RREQ, RREP and RERR) normalized by the total number of received data packets. It evaluates the efficiency of the routing protocol. 


\subsection{Simulation Results}

Fig.1 plots the packet delivery ratio versus traffic load for both AODV and LBBAODV protocols. Numerical results reveal that LBB-AODV achieves a larger packet delivery ratio compared to AODV, especially when the traffic load increases. This is because LBB-AODV chooses the least congested route for data transmission, which indirectly increases the overall network throughput. On the other hand, AODV without load-balancing mechanism suffers the high probability of the route failure and the data retransmission as the network traffic load becomes larger.

As can be seen in Fig.2, LBB-ADOV outperforms AODV in terms of average endto-end delay. The largest fall of average end-to-end delay is up to $17.3 \%$. Delay is mainly due to queuing in heavily loaded buffers. Even though the route path may be longer than that of AODV, LBB-ADOV tries to route packets along a less congested path to avoid overloading nodes, thereby minimizing path congestion and in turn reducing the overall queuing delay.

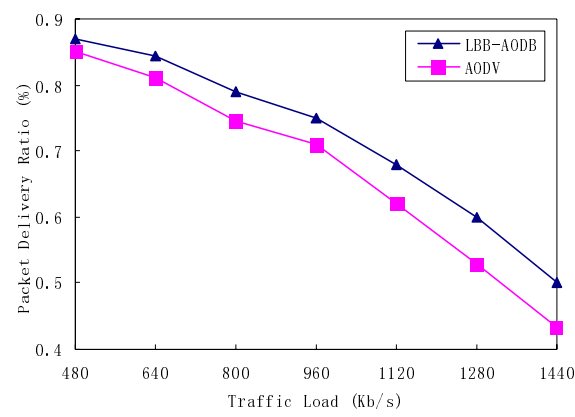

Fig. 1. Packet delivery ratio

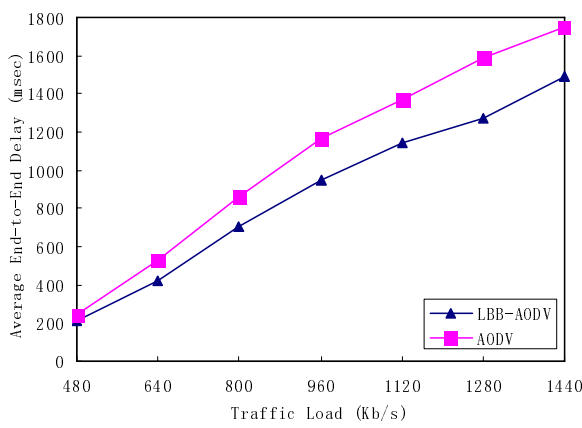

Fig. 2. Average end-to-end delay

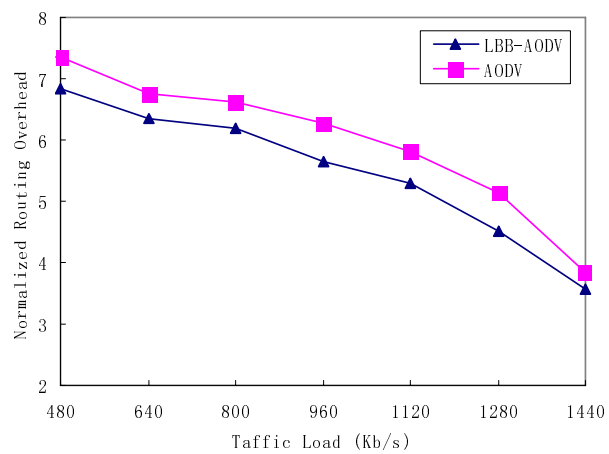

Fig. 3. Normalized routing overhead

In Fig.3, LBB-AODV demonstrates up to $16.2 \%$ reduction of the routing overhead against AODV. This is mainly due to the suppression of forwarding RREQs at the intermediate nodes, as the forwarding RREQs make up about $90 \%$ of the routing 
packets in AODV [8]. Because LBB-AODV prevents those over-loaded nodes from forwarding RREQs, the overall routing overhead is dramatically decreased. Moreover, LBB-AODV can establish routes more stable than AODV. As a result, LBB-AODV reduces link breakage and routing overhead, which is mainly caused by the route reconstruction. When the traffic load increases, the overall normalized routing overhead of both protocols decreases because of the source can send more data packets to its corresponding destination by using the discovered route.

\section{Conclusions}

In this paper, we proposed the load balance mechanism for the conventional AODV by considering the available bandwidth at each node. According to the local bandwidth status, each node has the right to re-broadcast the RREQ message in order to avoid network congestion and RREQ broadcast storm. Beside that, we also proposed that the RREQ message should contain an additional field to allow nodes to exchange the route bandwidth information among themselves. Moreover, we proposed a simple and effective bandwidth estimation approach based on the perceived bandwidth consumption of a node and its neighboring nodes.

Numerical simulations reveal that LBB-AODV significantly improves the packet delivery ratio and reduces the average end-to-end latency as well as the routing overhead in the presence of large traffic volume. The new scheme successfully balances the network load among nodes, and it can easily be incorporated in the existing on-demand routing protocol.

To facilitate practical implementation of our proposal, further research is required to investigate the optimal parameter under different network settings for LBB-AODV. Furthermore, we will look into how the proposed load balance mechanism can be incorporated in other on-demand routing protocols and make a fair comparison with other load balance schemes, like [3] and [4], in order to further improve the performance of LBB-AODV.

\section{Acknowledgement}

This work was supported in part by Shanghai Municipal R\&D Foundation under contracts 035107008, 04DZ15021-5A, 055115009, Shanghai Key Laboratory of Intelligent Information Processing (IIPL) and LG Electronics.

\section{References}

1. Y. Ganjali, A. Keshavarzian. Load balancing in ad hoc networks: single-path routing vs. multi-path routing. INFOCOM 2004. Twenty-third Annual Joint Conference of the IEEE Computer and Communications Societies Volume 2, Page(s):1120 - 1125 vol.2., March 2004.

2. D. Johnson and D. Maltz. Dynamic source routing in ad hoc wireless networks. Mobile Comput., pp. 153-181, 1996.

3. S. J. Lee, M. Gerla. Dynamic load-aware routing in ad hoc networks. Communications, 2001. ICC 2001. IEEE International Conference on Volume 10, Page(s):3206 - 3210 vol.10., June 2001. 
4. Y. J. Lee, G. F. Riley. A workload-based adaptive load-balancing technique for mobile ad hoc networks. Wireless Communications and Networking Conference, 2005 IEEE Volume 4, Page(s):2002 - 2007 Vol. 4., March 2005.

5. P. Mohapatra, J. Li, and C. Gui. QoS in mobile ad hoc networks, IEEE Wireless Commun. Mag. (Special Issue on QoS in Next-Generation Wireless Multimedia Communications Systems), pp. 44-52, 2003.

6. A. Nasipuri, R. Castaneda, and S. Das. Performance of multipath routing for on-demand protocols in ad hoc networks. ACM/Kluwer Mobile Networks and Applications (MONET) Journal, Vol. 6, No. 4, pp. 339-349, August 2001.

7. C. Perkins and E. Royer. Ad-Hoc On-Demand Distance Vector Routing (AODV). July 2003. RFC 3561.

8. C. Perkins, E. Royer, S. Das, M. Marina. Performance comparison of two on-demand routing protocols for ad hoc networks. Personal Communications, IEEE. Volume 8, Issue 1, Page(s):16 - 28., Feb. 2001.

9. D. D. Perkins, H. D. Hughes, C. B. Owen. Factors affecting the performance of ad hoc networks. Communications, 2002. ICC 2002. IEEE International Conference on Volume 4, Page(s):2048 - 2052 vol.4, May 2002.

10. P. Pham and S. Perreau, Multi-path routing protocol with load balancing policy in mobile ad hoc network. IFIP Int'l Conference on Mobile and Wireless Communications Networks (MWCN 2002), September 2002.

11. P. Pham and S. Perreau. Performance analysis of reactive shortest path and multi-path routing mechanism with load balance. IEEE Conference on Computer Communications (INFOCOM 2003), March 2003.

12. Liang Zhang, Yantai Shu, Yan Liuand Guanghong Wang, Adaptive Tuning of Distributed Coordination Function (DCF) in the IEEE 802.11 to Achieve Efficient Channel utilization. Future Telecommunication Conference 2003. Beijing, china, Dec. 2003.

13. Lingfang Zhang, Zenghua Zhao, Yantai Shu, Lei Wang, Yang, O.W.W. Load balancing of multipath source routing in ad hoc networks. Communications, 2002. ICC 2002. IEEE International Conference on Volume 5, Page(s):3197 - 3201 vol.5., May 2002.

14. Xiaofeng Zhong, Youzheng Wang, Shunliang Mi, Jing Wang. An Experimental Performance Study of Wireless ad hoc System Utilizing 802.11a Standard Base on different Routing Protocols. Asia Pacific Optical and Wireless Communications, October 2002. 